\title{
INTERPLAY OF PROBABILISTIC AND DETERMINISTIC INTERNAL LENGTHS IN SIMULATIONS OF CONCRETE FRACTURE
}

\author{
JAN ELIÁŠ ${ }^{*}$, JANA KADĚROVÁ* AND MIROSLAV VOŘECHOVSKÝ* \\ *Brno University of Technology \\ Brno, Czech Republic \\ e-mails: elias.j@fce.vutbr.cz, kaderova.j@fce.vutbr.cz,vorechovsky.m@fce.vutbr.cz
}

Key words: Probabilistic simulations, discrete model, three-point bending, internal length, correlation length

\begin{abstract}
The contribution presents simulations of fracture in concrete beams loaded in three-point bending via discrete model. The size and distribution of discrete units reflect the concrete heterogeneity and together with properties of their contacts provide an internal length scale. Due to a random placement of the discrete units, the model mimics natural randomness of material structure arising from its random heterogeneity. A second random component of the model considered is additional fluctuation of material parameters at contacts between the units. This randomness reflects variations in material properties due to mixing, drying, etc. and it is considered in a form of random field with its own internal length scale provided in a form of autocorrelation length.

By changing the ratios between the autocorrelation length and the internal length arising from heterogeneities, one can observe strong effects of this ratio on structural strength when cracks propagate from smooth surface. For deeply notched beams, the spatial strength fluctuations only affects the variance of the peak load and no significant effect observed on its mean value. The described dependency of structural strength on the ratio between the two characteristic lengths is demonstrated and elucidated.
\end{abstract}

\section{INTRODUCTION}

Concrete is recognized as highly heterogeneous material. The macroscopic material response and fracture behavior is influenced by the internal structure which can be reflected in the model by some internal length parameter. This is usually the case of continuum models, such as the Crack Band Model [1] or Nonlocal model [2]. A convenient way is to use discrete meso-scale models that contain information about the concrete internal structure directly. There are several types of such models developed for different purposes [3, 4, 5, 6]. The main advantages of the discrete meso-scale models are the natural discrete representation of cracks, the ability of capturing transition from distributed to localized fracture, automatic cracking in directions perpendicular to maximum principal stress, simpler formulation of constitutive relation using vectors instead of tensors, etc. This contribution utilizes a simplified version [7] of the lattice discrete particle model developed in [8, 9, 10].

Despite the rich information about material mesostructure, the meso-scale models are incomplete unless they include also spatial variability in material properties that arise during the production process (mixing, drying, etc.) and service life. These spatial fluctuations are usually modeled via random fields [11, 12, 13]. Even though there are several possible sources of random fluctuations, it is considered here that 
all of them can be approximately described by a single random field. This random field has its own characteristic length scale provided in a form of correlation length [14, 15]. Incorporation of the random fluctuations into the present discrete model is closely described in [16, 17].

The interplay between the characteristic length of the discrete system (called here deterministic internal length, $l_{\mathrm{d}}$ ) and the random field (autocorrelation length, $l_{\rho}$ ), is studied in the present contribution. Simulations of three-point bending beams, with and without a notch, are calculated using the discrete model enhanced with random fields (referred to as the probabilistic discrete model from here on). The simulations are performed repeatedly with randomly generated aggregate structure and with different realizations of the random field. A strong dependence of the mean value and standard deviation of the peak load on correlation length of the random field is reported.

\section{PROBABILISTIC DISCRETE MODEL}

The model at hand has two fundamental components. The first one is the accurate mechanics that provides stress redistribution, transition from distributed to localized crack and all other features necessary to capture properly the phenomena involved in concrete fracture. This includes also development and propagation of the Fracture Process Zone (FPZ), that is a standard name for a region around a crack tip that encloses material with nonlinear behavior. The model is a simplified version of [9], the full formulation accounts also e.g. for confinement. Our model is static, the solution proceeds in steps, iteration are performed to achieve static equilibrium at the end of each step. The location and size of the discrete units are randomly generated by computer based on sieve curve and on the total aggregate volume. The discrete units are ideally rigid; there is a constitutive relationship defined on contacts between them in terms of one normal and two tangential displacement jumps. The forces resulting from constitutive relationship are all dependent on a single damage parameter calculated from relatively complex set of equations (see [9]) that are based on several material parameters among which the most important are the strengths in tension and shear, $f_{\mathrm{t}}$ and $f_{\mathrm{s}}$, and fracture energies in tension and shear, $G_{\mathrm{t}}$ and $G_{\mathrm{s}}$.

The described model itself provides random response due to the randomness in the positions and sizes of the discrete units; because of the random meso-structure. However, we will refer the model without random fluctuations of material parameters to as the deterministic model from now on, and the mean value and standard deviation of the peak load provided by deterministic model will be denoted $\mu_{\mathrm{d}}$ and $\delta_{\mathrm{d}}$.

The second fundamental component is randomization of material parameters. The deterministic model combined with the randomization will be called the probabilistic model, and the corresponding mean and standard deviation of the peak load will be denoted $\mu_{\mathrm{p}}$ and $\delta_{\mathrm{p}}$, respectively.

In the probabilistic model, the material parameters governing fracture behavior $\left(f_{\mathrm{t}}, f_{\mathrm{s}}, G_{\mathrm{t}}\right.$ and $G_{\mathrm{s}}$ ) were randomized using random field. The correlation structure of the field was assumed to obey square exponential function and probabilistic distribution $H$ at any location inside the field was described by Weibull-Gauss juncture distribution function [18, 19] with the mean value $\mu_{h}$ and standard deviation $\delta_{h}$. Detailed description of the probabilistic model can be found in [17].

Two alternatives of varying the fracture energies were explored here. In the first one, all of the four random variables were considered linearly positively dependent. Only one random field ( $\boldsymbol{H}$, with the mean value $\mu_{h}=1$ ) therefore needs to be generated and random fields for fracture parameters are simply linear multiplies of $\boldsymbol{H}$. Denoting the mean values of the four random variables $\overline{f_{\mathrm{t}}}, \overline{f_{\mathrm{s}}}, \overline{G_{\mathrm{t}}}$ and $\overline{G_{\mathrm{s}}}$, one can obtain random field for these variables simply by

$$
\boldsymbol{H}_{X}=\bar{X} \boldsymbol{H}
$$

where $X$ stands for chosen random parameter. A feature noticeable with this alternative is that the Irwin characteristic length of the material is 
random. Such a length can be estimated at position $\boldsymbol{x}$, that has a random filed value $h(\boldsymbol{x})$, by

$$
l_{\mathrm{d}}(\boldsymbol{x})=\frac{E G_{\mathrm{t}}(\boldsymbol{x})}{\left[f_{\mathrm{t}}(\boldsymbol{x})\right]^{2}}=\frac{E \overline{G_{\mathrm{t}}} h(\boldsymbol{x})}{\left[\overline{f_{\mathrm{t}}} h(\boldsymbol{x})\right]^{2}}=\frac{\overline{l_{\mathrm{d}}}}{h(\boldsymbol{x})}
$$

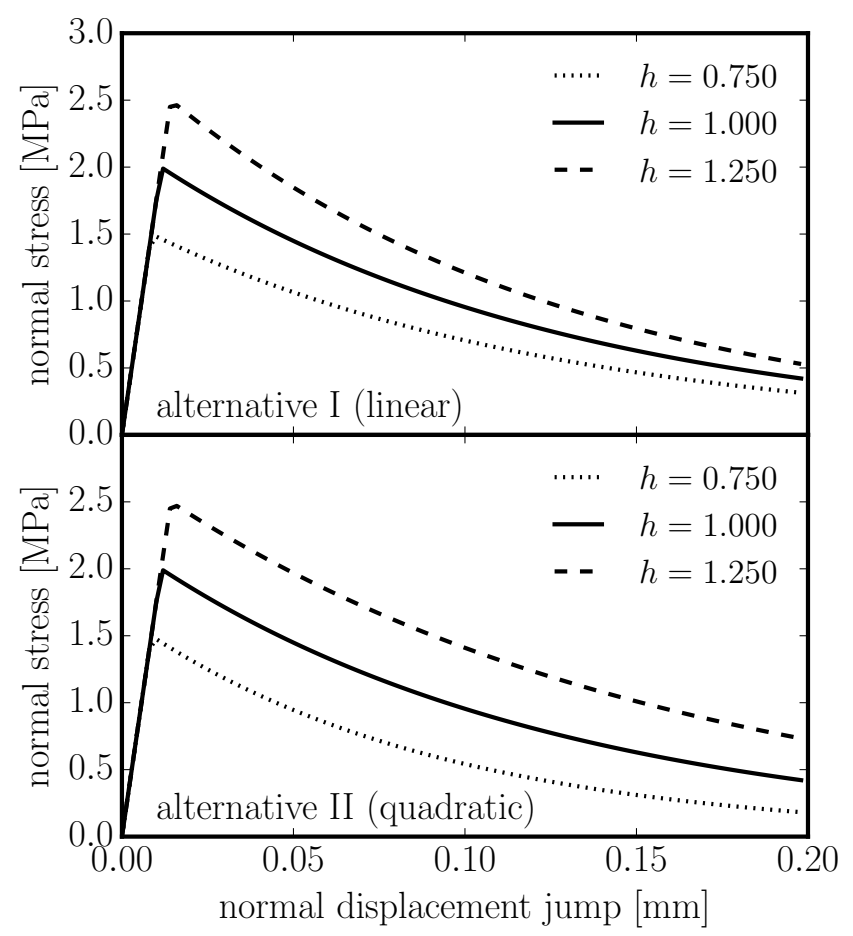

Figure 1: Constitutive law in monotonic normal loading in tension for both described alternatives.

\subsection{Randomization with a constant charac- teristic length}

Even though the alternative with variable characteristic length might be applied in general, for our purpose the second alternative where characteristic length is kept constant is preferred. Tensile and shear strengths are kept fluctuating according to Eq. 11, and the fracture energies in tension and shear follow

$$
\boldsymbol{H}_{X}=\bar{X}[\boldsymbol{H}]^{2}
$$

As a result, $\mathrm{Eq} 2$ is transformed into

$$
l_{\mathrm{d}}(\boldsymbol{x})=\frac{E G_{\mathrm{t}}(\boldsymbol{x})}{\left[f_{\mathrm{t}}(\boldsymbol{x})\right]^{2}}=\frac{E \overline{G_{\mathrm{t}}} h(\boldsymbol{x})^{2}}{\left[\overline{f_{\mathrm{t}}} h(\boldsymbol{x})\right]^{2}}=\overline{l_{\mathrm{d}}}
$$

Due to the squared value of random field in Eq. 3, the fracture energies are, on average, higher by a factor $\mu_{h^{2}}=\delta_{h}^{2}+\mu_{h}^{2}=\delta_{h}^{2}+1$. For purpose of this paper, it was assumed that $\delta_{h}=0.14$. Thus, fracture energies in alternative II are higher on average by $1.96 \%$ compared to the deterministic model or alternative $\mathrm{I}-\mathrm{a}$ fact that we ignore.

Both alternatives are depicted in Fig. 1. where the response of a single contact loaded monotonically in normal direction is plotted for different values of $h$. In alternative I, the $d e$ teministic internal length decreases with an increase in $h$ and the contact becomes more brittle.

Imagine application of a constant value of $h$ on the whole structure. Such a situation actually corresponds to one extreme case considered later, the case of infinitely large autocorrelation length $l_{\rho} \rightarrow \infty$. In such a case, alternative II leads simply to multiplication of structural strength by $h$ because the mechanics is independent of the chosen constant $h$. On contrary, alternative I produces nonlinear dependence on $h$ as the redistribution of stress in the mechanical system is affected by $h$. This is demonstrated in Fig. 2 including linear approximation line for $\mu_{\mathrm{d}}=26.5 \mathrm{kN}$. Alternative II is selected for further analyses as it enables easier interpretation of the results with randomization.

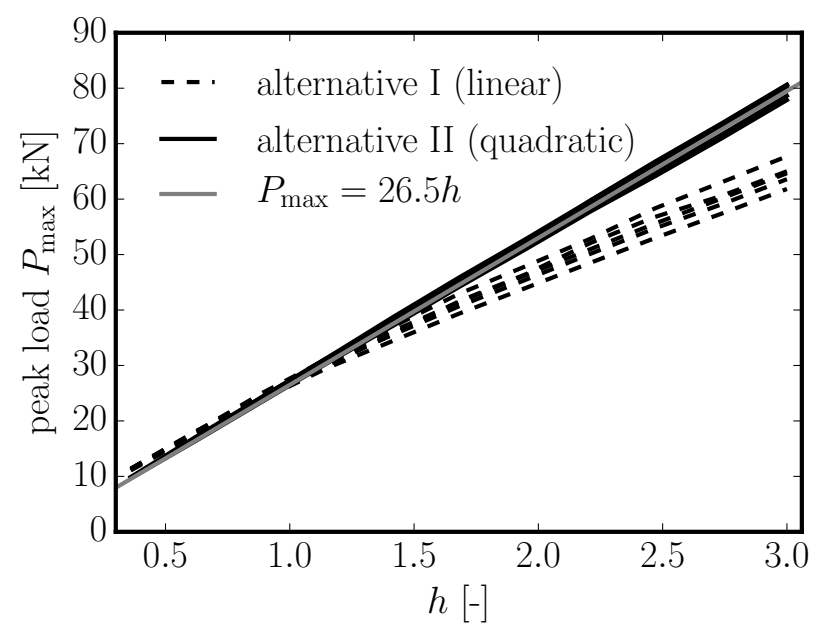

Figure 2: Peak load measured on several models with different meso-structure and with constant $h$ value for all contacts, two studied alternatives.

Since alternative II produces results that are just multiplied by a random factor $h$ that is con- 
stant over the whole structure, one can simply calculate what should theoretically be the mean and the standard deviation of the peak load of the probabilistic model in case of infinitely large correlation length. The product of two independent processes leads to simple formulas for the mean and standard deviation of the peak loads

$$
\begin{aligned}
\mu_{\mathrm{p}, \mathrm{t}} & =\mu_{\mathrm{d}} \mu_{h}=\mu_{\mathrm{d}} \\
\delta_{\mathrm{p}, \mathrm{t}} & =\sqrt{\delta_{\mathrm{d}}^{2} \delta_{h}^{2}+\mu_{\mathrm{d}}^{2} \delta_{h}^{2}+\delta_{\mathrm{d}}^{2} \mu_{h}^{2}}= \\
& =\sqrt{\delta_{\mathrm{d}}^{2} \delta_{h}^{2}+\mu_{\mathrm{d}}^{2} \delta_{h}^{2}+\delta_{\mathrm{d}}^{2}}
\end{aligned}
$$

\subsection{Material parameters}

Material parameters of the mechanical model and its probabilistic extension are partly identified and partly fabricated. The identification was based on experimental campaign described in [20] and it is briefly explained in [21]. The same material parameters were used also in this study including the $10 \mathrm{~mm}$ maximal diameter of grains. The width of FPZ should be, in such a case, $20-30 \mathrm{~mm}$.

The major focus is now on the effect of autocorrelation length, which was varied in the range $0-\infty$. When $\infty$, all of the contacts share an identical random value of $h$; when 0 , all contacts have random and independent values of $h$; when in between, a sample of random field with corresponding correlation length is generated and applied to $h$.
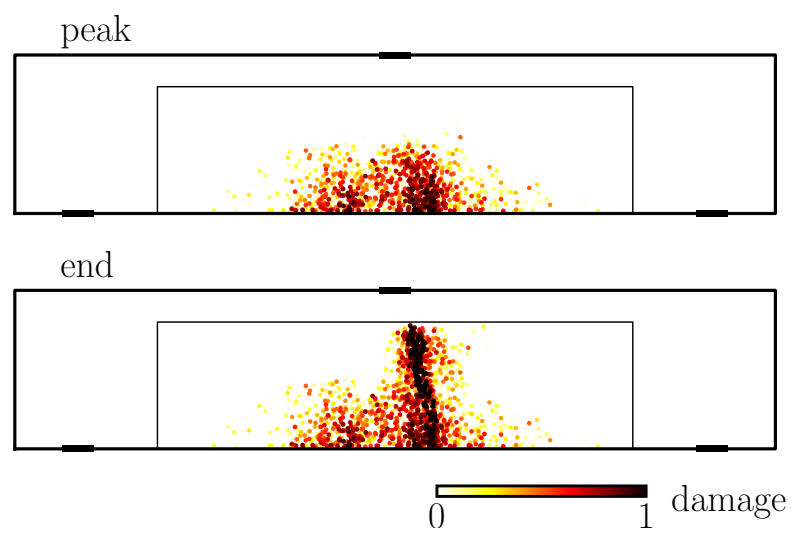

Figure 3: Distribution of damage at contacts of one realization of the probabilistic model at the peak load (top) and at the termination of the simulation (bottom) of unnotched beam.

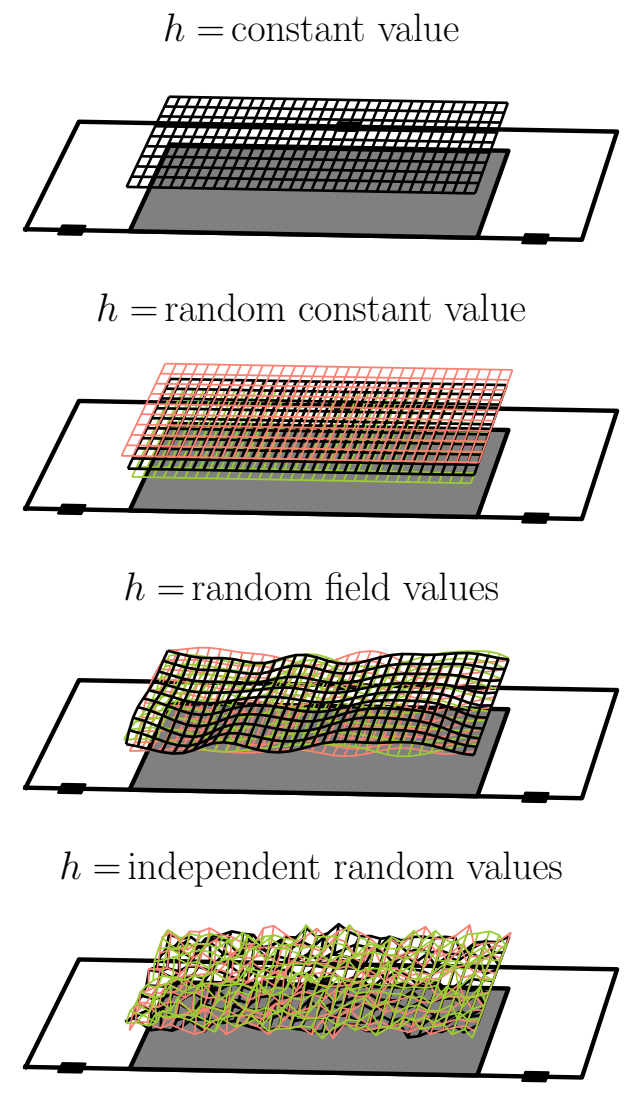

Figure 4: Values of the $h(\boldsymbol{x})$ parameter in the deterministic model (top) and the probabilistic models with correlation lengths $l_{\rho} \in\{\infty, 25,0\} \mathrm{mm}$ (a 2D illustration).

\section{STATISTICAL CHARACTERISTICS OF THE PEAK LOAD}

The simulated specimen was a concrete beam loaded in three-point bending. The dimensions of the specimen were: depth $150 \mathrm{~mm}$, length $720 \mathrm{~mm}$, span $600 \mathrm{~mm}$ and thickness $40 \mathrm{~mm}$. Two configurations were considered - without a notch and with a central notch of length $75 \mathrm{~mm}$. Only the central part of the beam was represented by the discrete model, the surrounding material was modeled by linear elastic finite elements.

The peak load of such a beam was calculated using both the deterministic and probabilistics model with several autocorrelation lengths. To estimate the mean value and standard deviation of the peak load, 100 realizations of every model variant was performed. The realizations differed by random meso-level arrangement and 

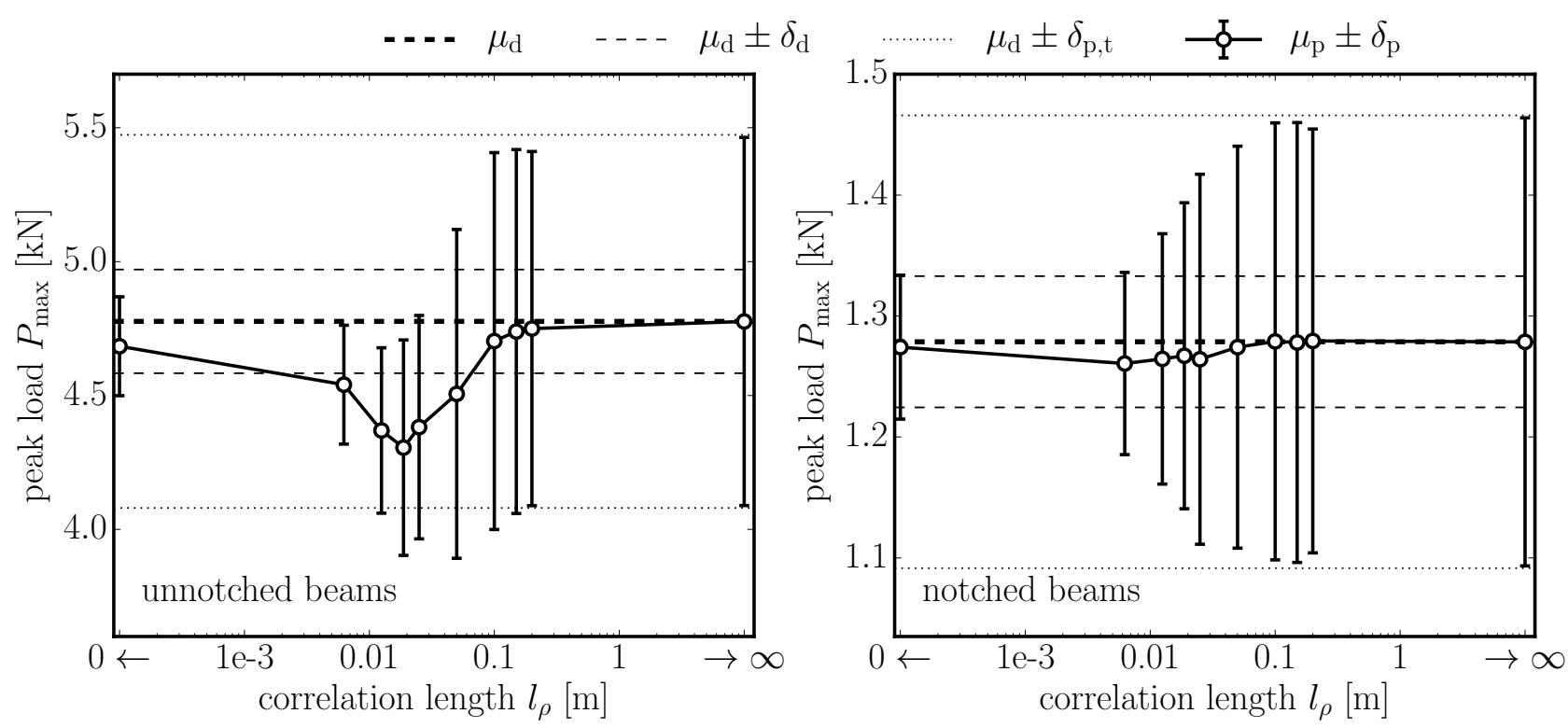

Figure 5: Mean value and standard deviation of peak load computed on unnotched (left) and notched (right) beams using deterministic model $\left(\mu_{\mathrm{d}}, \delta_{\mathrm{d}}\right)$ and probabilistic model $\left(\left(\mu_{\mathrm{d}}, \delta_{\mathrm{d}}\right)\right)$. Theoretical standard deviation for $l_{\rho} \rightarrow \infty$ according to Eq. 6 is denoted $\delta_{\mathrm{p}, \mathrm{t}}$.

random field realization if included. The distribution of damage in one realization of the probabilistic model with $l_{\rho}=25 \mathrm{~mm}$ is shown in Fig. 3. Three realizations of the random field in selected variants of the model are sketched in Fig. 4.

The resulting mean values and standard deviations of the peak loads are presented in Fig. 5 . On the right hand side, the results of unnotched configuration are shown. The mean values of probabilistic and deterministic models are almost identical irrespectively of the correlation length. This is due to the strong stress concentration at the notch tip; the crack always starts from that location. And even in randomized model, the region around the notch tip keeps the mean random value $\mu_{h}=1$. However, the standard deviation exhibits a strong dependence. For large correlation length, it obeys assumption of independent sources of variance from (i) the random field scaling and, (ii) the randomness due to meso-structure (as derived in Eq. 6). As the correlation length $l_{\rho}$ decreases, it decreases towards $\delta_{\mathrm{d}}$. The decrease in standard deviation is caused by a finite size of FPZ. Failure of the beam is always triggered by damaging many contacts within an FPZ. Simultane- ous failure of many contacts leads to averaging of contact strengths within FPZ. For very short autocorrelation length, the the effect of spatial variability within FPZ gets averaged out and the peak loads are almost identical to the deterministic simulations.

The same averaging effect is responsible for the decrease in standard deviation in the case of unnotched beam, see Fig. 5 left hand side. Contrary to presence of strong stress concentration that dictates crack location, in this case the crack may initiate anywhere along the bottom surface. The peak load is reached, again, only after damaging a certain material volume, FPZ. As the correlation length decreases, the fluctuations are more frequent and the lower FPZ strength may appear. However, when the correlation length drops below the FPZ size, the averaging effect is responsible for increase of its the average FPZ strength.

\section{SIZE AND SHAPE OF THE FPZ}

The results from numerical simulations can also provide some information about the FPZ in terms of its size and its shape. These two characteristics could be investigated by displaying the energy dissipation density. The particu- 

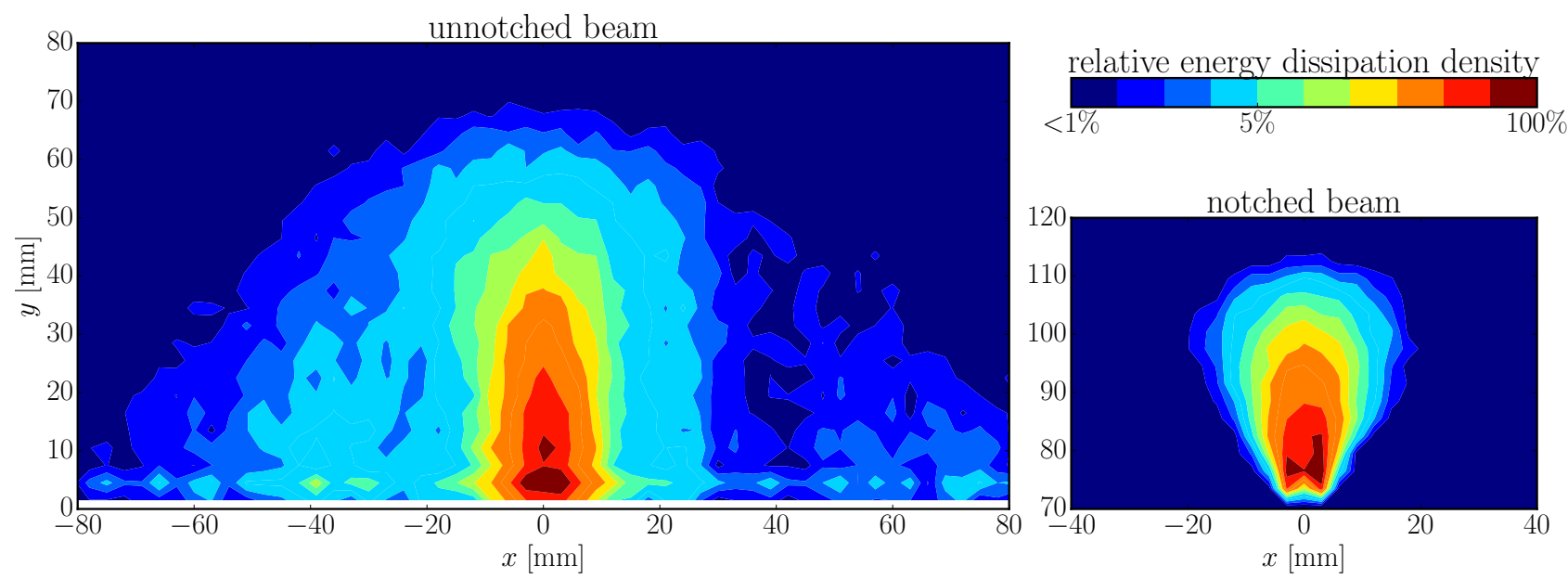

Figure 6: Relative energy dissipation density released within the solution step in which the peak load was reached; $l_{\rho}=25 \mathrm{~mm}$. Left: unnotched beam, right: notched beam.

lar interest is in the FPZ at the peak load, since it can identify material volume responsible for magnitude of the peak load.

The energy dissipated at the contacts within the computational step right prior to the peak load was normalized to provide unit sum. Then, this data from all 100 models was summed up into the bins of size thickness $\times 3 \times 3 \mathrm{~mm}$ organized in a regular grid. The bin into which the dissipating contact contributed was determined based on the location of the contact midpoint. In case of unnotched beams, the location of the final crack is varying. The macrocrack location was estimated as a centroid of the energy released in the final simulation step in the boundary layer. An appropriate bin was then found based on relative location of the dissipating contact with respect to the macrocrack location.

Fig.6 shows 2D plot of the relative energy dissipation density in logarithmic scale for $l_{\rho}=$ $25 \mathrm{~mm}$. The red color marks areas with the highest value (set to $100 \%$ in both beams), the dark blue color shows bins with less than $1 \%$ of the energy dissipation density compared to the maximum.

The figures are comparable under the assumption that when the structure reaches the peak load, the maximal rate of energy dissipation density is the same irrespectively of the size and boundary conditions. Assuming this statement valid, we can compare now the FPZs. Tak- ing the contour at the level $5 \%$ as the boundary of FPZ, one can see that both the width and depth of the FPZ are slightly larger for unnotched beam. Its width and depth is approximately $30 \mathrm{~mm}(20 \mathrm{~mm})$ and $50 \mathrm{~mm}(35 \mathrm{~mm})$ for unnotched (notched) beam, respectively.

\section{EXTREMES OF MOVING AVERAGES OF 1D RANDOM FIELDS}

In this section, an analogy between the probabilistic simulations of unnotched beams and properties of moving averages of random fields is elucidated. As already mentioned above, the average peak load for infinitely large autocorrelation length is equal to the average results obtained with the deterministic model. On the other hand, results obtained with a "white noise", i.e. with autocorrelation length below the size of aggregates also seem to tend, on average, to the deterministic results. However, there is a certain range of autocorrelation lengths of the local strength parameters that lead to a drop in the average peak load.

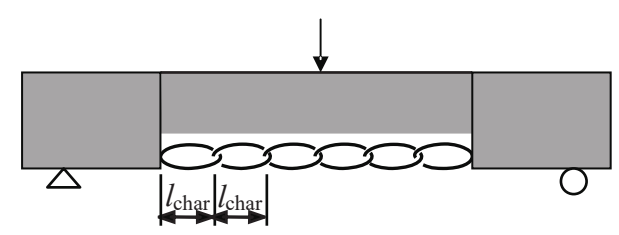

Figure 8: Idealization of the beam by a chain of sizeable potential fracture process zones. 

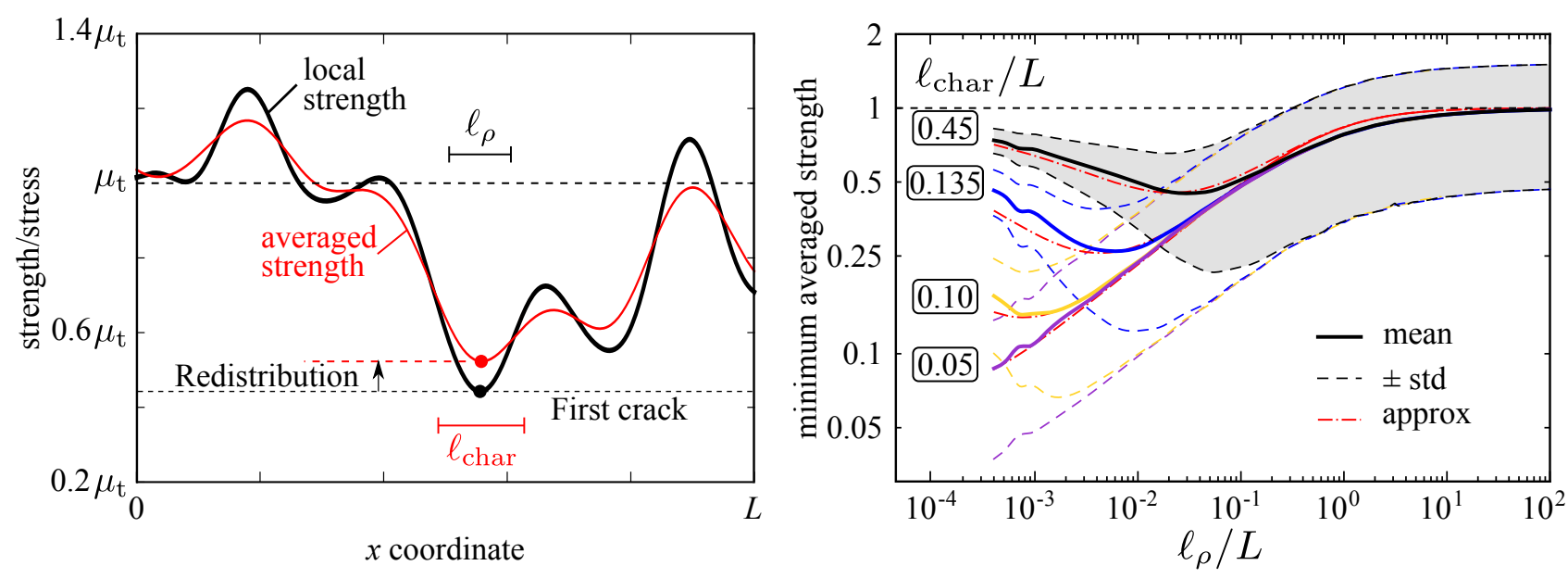

Figure 7: Left: Illustration of a moving average of a realization of 1D process of local tensile strength. Right: Dependence of the extreme (minimum) of the random field as a function of the relative chain length $\left(l_{\rho} / L\right)$ and averaging support $\left(l_{\text {char }} / L\right)$.

Intuitively, one can explain this feature by the argument that when the size of the locally weak region (size of about one autocorrelation length) reaches approximately the size of FPZ, the peak load will reach its minimum, on average. The reason is that the weak FPZ that must develop to reach the peak load, and it can select the weakest spot along the bottom surface of the beam. This feature is depicted in Fig. 8 .

In order to capture the essence of the feature and to quantify how deep the drop in the average peak load is, a simple model studied in [23] might suffice. Imagine a 1D structure (a chain) loaded in uniaxial tension. The chain has a spatially varying local strength described by a random field (a realization of such a process is the black line in Fig. 7 left). The chain fails only after a certain amount of material, mimicking the FPZ, fails. The extent of the FPZ is represented by its width which translates into length along the chain denoted as $l_{\text {char }}$ (see the short red line in Fig. 7 left). The length $l_{\text {char }}$ represents the material heterogeneity in the same sense and length $l_{\mathrm{d}}$ used above for the discrete model. Assume now that the local strength of the chain is a $1 \mathrm{D}$ process and it serves as a barrier to a stress field that increases from below. Since the tensile force is equal along the chain, the stress is a constant function. When the stress reaches the minimum of the local strength along the bar (a black dashed line in Fig. 7 left), the ability to redistribute stress to its surrounding can be mimicked by averaging the local strength along a region (support) $l_{\text {char }}$. The simplest way to perform a local average is to use a rectangular (constant) weight function with a support $l_{\text {char }}$. To find the strength of such a chain with redistribution, one has to seek the lowest point along the chain after performing the moving average. The result of this moving averaging is the red line in Fig. 7lleft. The small arrow in the figure marks the stress increase from the first cracking to the peak load. This increase is available thanks to local averaging. When the stress level reaches the short red dashed line, the minimum of a moving average is attained.

Therefore, to describe a strength of such a structure with redistribution, one can study the purely mathematical problem of distribution of a minimum of a moving average of the parent process that describes the local strength. When the chain length is fixed (e.g. a unit length $L=1$ ), the problem features interplay of two lengths: the autocorrelation length, $l_{\rho}$ and the averaging support, $l_{\text {char }}$. Strictly speaking, this setting is relevant to a problem of uniaxial tension and not the problem of three-point bending where the locally averaged tensile stress at the bottom of the bar is approximately linear. However, the shape of the dependence of the average and standard deviation of the peak load on the autocorrelation length is qualitatively obtained, 
see Fig. 7 right and compare it with Fig. 5 left.

Fig. 7 right is constructed for Weibull random strength field with a unit standard deviation. It studies the dependence of bar strength on $l_{\rho}$ with several different averaging lengths, $l_{\text {char }}$. When $l_{\text {char }} \rightarrow 0$, the problem reduces to the problem of extremes of Weibullian random fields studied previously in [24, 25]. When $l_{\text {char }} \rightarrow \infty$, the samples of random fields are random constant functions and the averaging does not modify the samples. However, a nonzero averaging length $l_{\text {char }}$ modifies the sample paths and it has been found [23] that the field paths represent, approximately, a different random field with an effective autocorrelation length $l_{\text {eff }}\left(l_{\text {char }}, l_{\rho}\right)=l_{\rho}\left(l_{\text {char }} / l^{\star}+l^{\star} /\left(l_{\text {char }}+\right.\right.$ $\left.l^{\star}\right)$ ), in which the cross-over correlation length $l^{\star} \approx 1.69 l_{\rho}$. The new process has also a different standard deviation which can be approximately transformed into effective Weibull modulus: $m_{\text {eff }}\left(m, l_{\text {char }}, l_{\rho}\right)=m\left(l_{\text {char }} / l^{\star}+l^{\star} /\left(l_{\text {char }}+\right.\right.$ $\left.\left.l^{\star}\right)\right)^{1 / 2}$. Now that the effective autocorrelation length and scatter is known, the minima have the mean value $\mu_{\mathrm{p}, \mathrm{t}}=\left(l_{\mathrm{eff}} /\left(l_{\mathrm{eff}}+l\right)+\right.$ $\left.1 / l_{\text {eff }}\right)^{-1.0 /\left(0.9 m_{\text {eff }}\right)}$. This formula is used to plot the dash-dot lines in Fig. 7 right. More details on this theory can be found in [23].

\section{CONCLUSIONS}

Probabilistic discrete meso-scale model was employed to simulate fracture of beams with and without notch. The effect of ratio between the autocorrelation length of the random field determining fluctuations of material parameters and the internal length of material arising from its meso-structure was studied. The results show the interplay between these two characteristic lengths.

In notched simulations, the average peak load is found to be independent of the correlation length. The reason for such insensitivity is that the crack is forced to propagate from one specific location (notch tip). However, the standard deviation decreases as the correlation length decreases due to averaging of the fluctuations within the fracture process zone.

In unnotched beams, the standard deviation follows the same trend. The mean value of peak load in models with both, very low and very high correlation lengths of random field, is approximately equal to the one obtained with models without random field. However, there is a certain range of correlation lengths for which the mean value of the peak load exhibits a clear downtrend by about $10 \%$. The minimum of the mean value occurs when the correlation length equals approximately the internal material length. Such a correlation length enables the structure to sample the position of fracture process zone inside the weakest spot that has the size of the characteristic length. Decrease below such a correlation length leads to more averaging within the fracture process zone which homogenizes the response. Increase in correlation length leads to smoothing of the local strength and prohibits the weakest spots to appear.

Generally, for smooth stress fields (unnotched specimens), the main features obtained using the presented probabilistic discrete model can essentially be captured by the behavior of moving averages of random fields. Local averaging mimics the stress redistribution within the fracture process zone and this averaging interacts with the autocorrelation length of the strength random field.

Based on the simulation results, comparison of the FPZ size at the peak load was done for the notched and unnotched beam geometries. It was found that the FPZ is slightly wider and deeper in beams without a notch. The FPZ width is in agreement with generally accepted assumption of approximately 3 maximum aggregate diameters.

\section{ACKNOWLEDGEMENT}

The authors acknowledge financial support obtained through the Czech Science Foundation projects nos. GA13-03662S and GA1519865Y.

\section{References}

[1] Z.P. Bažant and B.H. Oh. Crack band theory for fracture of concrete. Matériaux et 
Construction, 16(3):155-177, 1983.

[2] M. Jirásek. Nonlocal models for damage and fracture: Comparison of approaches. International Journal of Solids and Structures, 35(3132):4133 - 4145, 1998.

[3] J. E. Bolander and S. Saito. Fracture analyses using spring networks with random geometry. Engineering Fracture Mechanics, 61:1569-591, 1998.

[4] E. Schlangen and E.J. Garboczi. Fracture simulations of concrete using lattice models: Computational aspects. Engineering Fracture Mechanics, 57(2-3):319332, 1997.

[5] H.-K. Man and J.G.M. van Mier. Influence of particle density on 3D size effects in the fracture of (numerical) concrete. Mechanics of Materials, 40(6):470 486, 2008.

[6] A. G. D'Addetta, F. Kun, and E. Ramm. On the application of a discrete model to the fracture process of cohesive granular materials. Granular Matter, 4(2):77-90, 2002.

[7] J. Eliáš and Z. P. Bažant. Fracturing in concrete via lattice-particle model. In E. Oñate and D. Owen, editors, 2nd international conference on particle-based methods - fundamentals and applications, Barcelona, Spain, 2011.

[8] G. Cusatis, Z.P. Bažant, and L. Cedolin. Confinement-shear lattice CSL model for fracture propagation in concrete. Computer Methods in Applied Mechanics and Engineering, 195(52):7154-7171, 2006.

[9] G. Cusatis and L. Cedolin. Two-scale study of concrete fracturing behavior. Engineering Fracture Mechanics, 74(12):317, 2007.

[10] G. Cusatis, D. Pelessone, and A. Mencarelli. Lattice discrete particle model
(LDPM) for failure behavior of concrete. I: Theory. Cement and Concrete Composites, 33(9):881-890, 2011.

[11] M. Vořechovský and V. Sadílek. Computational modeling of size effects in concrete specimens under uniaxial tension. Internationa Journal of Fracture, 154(12):27-49, 2008.

[12] P. Grassl and Z. P. Bažant. Random latticeparticle simulation of statistical size effect in quasi-brittle structures failing at crack initiation. Journal of Engineering Mechanics - ASCE, 135:85-92, 2009.

[13] M. Georgioudakis, G. Stefanou, and M. Papadrakakis. Stochastic failure analysis of structures with softening materials. Engineering Structures, 61:13 - 21, 2014.

[14] M. Vořechovský. Interplay of size effects in concrete specimens under tension studied via computational stochastic fracture mechanics. International Journal of Solids and Structures (Elsevier), 44(9):2715-2731, 2007.

[15] Z. P. Bažant, M. Vořechovský, and D. Novák. Asymptotic prediction of energetic-statistical size effect from deterministic finite element solutions. Journal of Engineering Mechanics (ASCE), 133(2):153-162, 2007.

[16] J. Eliáš, M. Vořechovský, and Z. P. Bažant. Stochastic lattice simulations of flexural failure in concrete beams. In $8 t h$ International Conference on Fracture Mechanics of Concrete and Concrete Structures, Toledo, Spain, 2013.

[17] J. Eliáš, M. Vořechovský, J. Skoček, and Z.P. Bažant. Stochastic discrete mesoscale simulations of concrete fracture: comparison to experimental data. Engineering Fracture Mechanics, 135:1-16, 2015. 
[18] Z.P. Bažant and S.-D. Pang. Activation energy based extreme value statistics and size effect in brittle and quasibrittle fracture. Journal of Mechanics and Physics of Solid, 55(1):91-131, 2007.

[19] J.-L. Le, Z.P. Bažant, and M.Z. Bazant. Unified nano-mechanics based probabilistic theory of quasibrittle and brittle structures: I. strength, static crack growth, lifetime and scaling. Journal of Mechanics and Physics of Solid, 59(7):1291-1321, 2011.

[20] D. Grégoire, L.B. Rojas-Solano, and G. Pijaudier-Cabot. Failure and size effect for notched and unnotched concrete beams. International Journal for Numerical and Analytical Methods in Geomechanics, 37(10):1434-1452, 2013.

[21] J. Kaděrová and J. Eliáš. Simulations of bending experiments of concrete beams by stochastic discrete model. Key Engineering Materials, 627:457-460, 2015.

[22] M. Vořechovský and D. Novák. Model- ing statistical size effect in concrete by the extreme value theory. In J. Walraven, J. Blaauwendaad, T. Scarpas, and B. Snijder, editors, $5^{\text {th }}$ International Ph.D. Symposium in Civil Engineering, held in Delft, The Netherlands, volume 2, pages 867875, London, UK, 2004. A.A. Balkema Publishers.

[23] M. Vořechovský and V. Sadílek. Extremes and moving averages of random fields with application to strength of materials. Probabilistic Engineering Mechanics, page in preparation, 2016.

[24] M. Vořechovský and R. Chudoba. Stochastic modeling of multi-filament yarns: II. Random properties over the length and size effect. International Journal of Solids and Structures (Elsevier), 43(3-4):435-458, 2006.

[25] M. Vořechovský. Incorporation of statistical length scale into Weibull strength theory for composites. Composite Structures, 92(9):2027-2034, 2010. 